Neurosurg Focus 9 (6):Article 2, 2000

\title{
Cytokine immunogene therapy
}

\author{
Roberta P. Glick, M.D., Terry Lichtor, M.D., Ph.D., And Edward P. Cohen, M.D. \\ Departments of Neurosurgery, Anatomy and Cell Biology, Rush Medical College, Cook County \\ Hospital and University of Illinois; Department of Neurological Surgery, Rush Medical College, \\ Loyola University Chicago, and Cook County Hospital; and Department of Microbiology and \\ Immunology, University of Illinois College of Medicine, University of Illinois at Chicago, Illinois
}

\begin{abstract}
The prognosis for patients with either a primary or metastatic brain tumor is poor. Clearly new forms of therapy to improve the long-term survival of patients with malignant brain tumors are urgently needed. The authors are in the process of developing a new and novel form of treatment for primary and metastatic brain tumors in which they use genes involved in growth repression. In particular most tumors fail to induce an antitumor immune response strong enough to kill the tumor. Under appropriate circumstances, however, immunity can be produced in unique structures on the tumor cells known as antigens. To prepare the vaccine, genes are transferred into a fibroblast cell line that causes the cell to produce cytokines, the potent proteins known to stimulate the immune system. These cells are subsequently injected into the tumor bed, resulting in the development of an antitumor immune response. In experiments described in this manuscript, the authors have investigated a number of ways of augmenting the immune response by administering this type of cellular vaccine. They found that mice with a primary intracerebral glioma, melanoma, or breast cancer treated with this allogeneic cytokine-secreting vaccine survived significantly longer than untreated mice. Additionally the vaccine was found to stimulate a systemic antitumor immune response, as shown by immunocytotoxic studies, histopathological examination, and delayed immune memory responses. In summary, these results indicate that immunogene therapy is a promising new targeted therapy for the treatment of intracerebral malignant tumors.
\end{abstract}

KEY WORDS - cytokine • gene therapy - interleukin-2 immunotherapy • breast cancer

The current prognosis for patients with malignant brain tumors remains poor. ${ }^{26}$ Malignant gliomas are the most common primary brain tumor. Despite the use of surgery, radiotherpy, and chemotherapy, the 2-year survival rate remains less than $20 \%$. Patients harboring malignant melanoma, one of the most common tumors to metastasize to the CNS, survive approximately 3 months after symptoms first occur. ${ }^{33}$ Of patients with malignant melanoma, 20 to $30 \%$ develop cerebral metastasis. Breast cancer is the second leading cause of cancer-related death in American women. In 1997, more than 40,000 women died of the disease. Brain metastases occur in 15 to $30 \%$ of patients with breast cancer. The 2-year survival for these patients, despite the provision of optimum conventional therapies, remain poor. Thus, new and innovative forms of effective

Abbreviations used in this paper: CNS = central nervous system; GM-CSF = granulocyte-macrophage-colony stimulating factor; IFN = interferon; IL = interleukin; MHC = major histocompatibility complex. treatments are urgently needed for both primary and metastatic malignant CNS tumors.

One emerging strategy in the treatment of tumors involves stimulation of an immunological response directed against the neoplastic cells. The hope is that the immune system can be called into play to destroy malignant cells. However, in most instances, proliferating tumors do not provoke antitumor cellular immune responses. The precise mechanisms that enable antigenic neoplasms to escape host immunity are incompletely understood. Although neoplastic cells form weakly immunogenic tumorassociated antigens, the cells appear to escape recognition by the immune system. Successful methods to confer immunity on tumor-associated antigens could lead to tumor cell destruction and to prolonging the survival of patients with cancer. A variety of strategies has been used to increase the antigenic properties of tumor cells. In some instances, objective evidence of tumor regression has been observed in patients receiving immunizations comprised only of tumor cell immunogens, suggesting the potential 
effectiveness of this type of immunotherapy for malignant neoplasms.

Some of the molecular techniques used by our laboratory and others to augment the immune response to tumor cells include: 1) genetic modification of cells to secrete immune-augmenting cytokines such as IL-2, GM-CSF, IFN, and IL-12; 2) genetic modification of the cell to express foreign (allogeneic) MHC determinants; 3) genetic modification of cells to express costimulatory molecules, such as B7, required for T cell activation; 4) genetic modification of cells to express tumor-associated antigens; 5) use of tissue-specific promoters; and 6) modification of delivery technique, as, for example, systemic compared with intracerebral immunization. We will explore these methods of augmenting the immune system.

\section{SYSTEMIC CYTOKINES}

Recent advances in our understanding of the biological nature of the immune system have led to the identification of numerous cytokines that modulate immune responses. ${ }^{5,10,11,21,25,31,35,38}$ These agents mediate many of the immune response involved in antitumor immunity. Several of these cytokines have been produced by recombinant DNA methodology and evaluated for their antitumor effects. Cytokines have been used in systemic therapy for tumors outside the CNS. In particular, systemic biological therapy IL-2 has been shown to cause significant antitumor effects in patients with advanced metastatic melanoma or renal cell cancer. ${ }^{34,36}$ Interleukin-2 has no direct toxic effect on cancer cells. Its antitumor activity is mediated by modulation of the host's immunological response to the neoplasm. Interleukin-2 is required for the growth of cytotoxic T lymphocytes (CD8+ cells), and it stimulates the cells to engage in cytotoxic activity. It enhances the activity of natural killer/lymphokine-activated killer cells, which possess antitumor activity, whereas the CD8+ cells provide antigen-specific antitumor functions. Activation of both of these cell types most likely is required for the optimum opportunity for tumor rejection. Another cytokine, IFN $\gamma$, induces the expression of MHC Class I determinants and augments the sensitivity of tumor cells to cytotoxic T lymphocyte-mediated lysis. Recently, attention has been given to IL-12, which activates antigenpresenting cells for antitumor rejection. Thus, the weakly antigenic neoplastic cells may become immunogenicstimulating antitumor immune responses as a result of the presence of these cytokines.

Use of IL-2 in the treatment of patients with high-grade gliomas has been actively pursued, particularly since it was observed that such patients may have both a decrease in IL-2 production and IL-2 receptor expression. ${ }^{37}$ However, significant antitumor effects have not been found when treating patients who harbor gliomas with cytokines. ${ }^{2,27,28,44}$ Immunotherapeutic attempts against gliomas have involved the systemic injection of intrathecal and intratumoral autologous lymphocytes and/or the administration of various cytokines that might enhance the immune response. $2,27,28,39,44$ Systemic administration of cytokines as well as lymphokine-activated killer cells has been attempted, but success has been limited..$^{2,27,28,44}$ Some of the failures, observed in the clinical trials in which pa- tients with gliomas received systemic IL-2 vaccinations, may be secondary to adverse effects of the therapy. Interleukin-2 causes an increase in vascular permeability that leads to accumulations of fluid in body compartments and possibly to intracerebral edema. Because the administration of IL-2 does not result in neutropenia or immunosuppression, patients are not susceptible to the opportunistic infections frequently observed following treatment with chemotherapeutic agents known to suppress the immune system. However, the CNS toxicity that commonly occurs with this form of treatment limits the amount of IL2 that can be administered. ${ }^{27}$ Interferon- $\gamma$ has been found to inhibit the in vitro growth of glioma cell lines. ${ }^{7}$ Many of the findings established using brain tumor cell lines have not been observed in vivo, and most of the in vivo studies involve the treatment of tumors in immunodeficient nude mice. ${ }^{45}$ The relevance of these models to human disease is uncertain.

\section{GENE THERAPY}

The use of gene therapy for the treatment of patients with malignant tumors provides a new method for the delivery of cytokines. Transduction of various cytokinespecifying genes into malignant cells as well as cytokine secretion by the cells themselves has also resulted in an augmentation of their immunogenic properties. Transfection of the human IFN $\gamma$ gene into human glioma cells has been shown to lead to an inhibition of growth when the tumor cells were exposed to lymphokine-activated killer cells. ${ }^{29}$ In a human glioma cell line significant inhibition of tumor growth in nude mice was observed when an IL-4-secreting cell line was implanted subcutaneously in addition to the glioma cells. ${ }^{45} \mathrm{~A}$ similar effect was also observed when the tumor and IL-4-secreting cells were injected intracerebrally. Rats injected subcutaneously with 9L gliosarcoma cells transduced with the $I L-2$ gene demonstrated tumor eradication, but only minimal effects were seen when the cells were injected intracerebrally. ${ }^{32}$ In other studies it was found that GM-CSF-transduced nonreplicating (irradiated) melanoma cells administered as a subcutaneous vaccine, but not following an intracranial injection, conferred some protection from intracranial challenge with wild-type melanoma. ${ }^{41}$ In contrast, the direct intracranial injection of nonreplicating (irradiated) melanoma cells secreting IL-2 was protective, whereas injection into one flank with IL-2-producing cells was not. However, a synergistic antimelanoma response was achieved in the brains of mice harboring melanoma when the subcutaneous injection of nonreplicating melanoma cells genetically engineered to secrete GM-CSF as a vaccine was combined with the local administration of IL-2. ${ }^{41}$ These findings provide a basis for the application of cytokine delivery to brain tumors both as a systemic vaccine and/or via local administration.

Genetic engineering techniques can also be used to deliver cytokines to an intracranial tumor, and this method involves the use of replication-competent herpes simplex viruses. ${ }^{1}$ In recent studies, it was reported that a herpes simplex virus modified for the expression of IL-4 resulted in a significant prolongation of survival in mice harboring intracranial glioma. Furthermore, an inflammatory reac- 


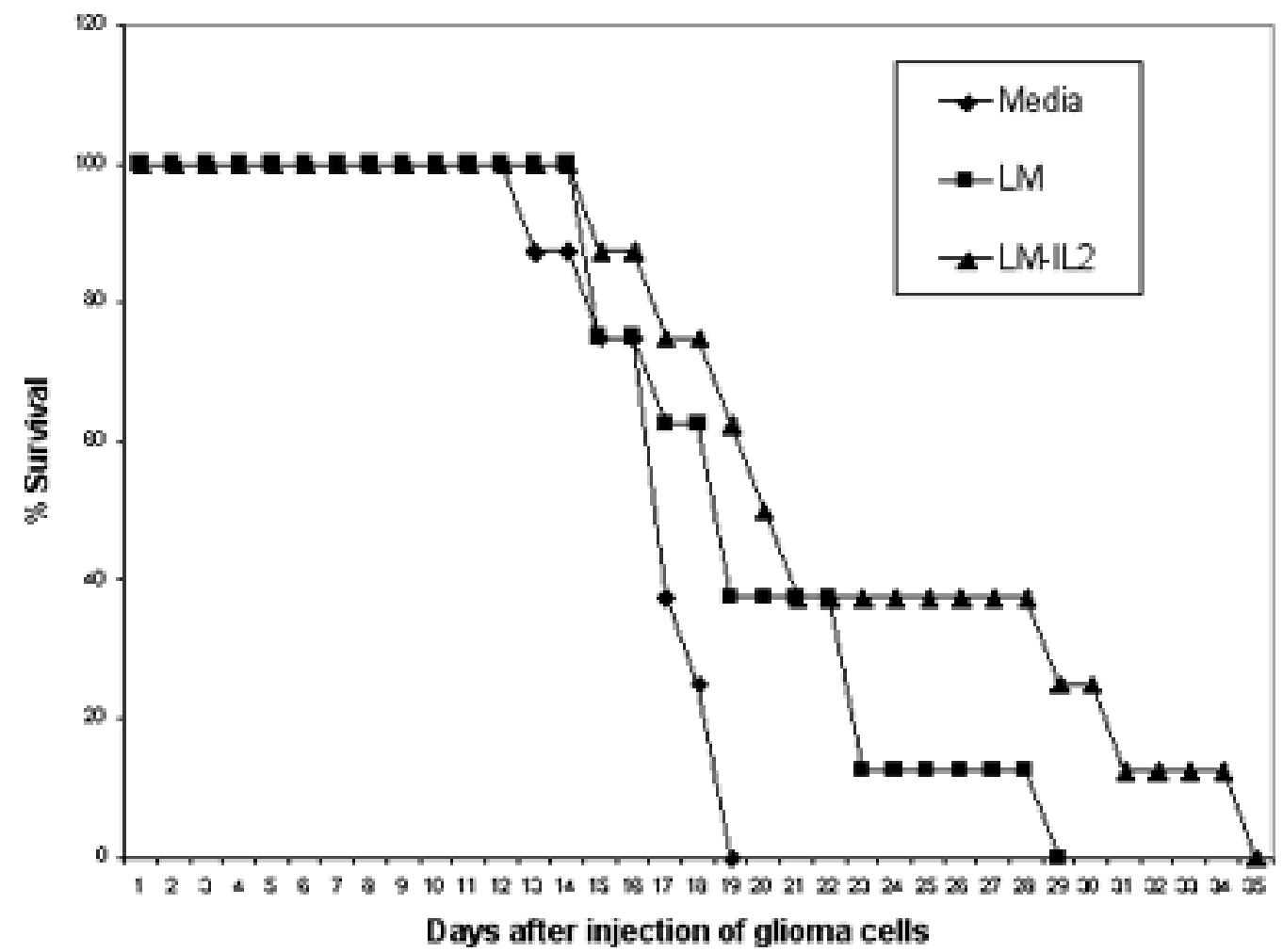

Fig. 1. Graph showing the survival rate of mice receiving an intracerebral injection of glioma cells and fibroblasts (LM cells) engineered to secrete cytokines. The $\mathrm{C} 57 \mathrm{Bl} / 6$ mice (eight per group) were injected intracerebrally with a mixture of $10^{6}$ cells of one of the cell types and $10^{5} \mathrm{Gl} 261$ glioma cells. The median lengths of survival were as follows (in days; mean \pm standard deviation): mice with nonimmunized glioma cells, $16.9 \pm 1.9$; glioma plus fibroblast cells, $20 \pm 4.5$; and glioma plus fibroblast and IL-2 (LM-IL-2) cells, $23.4 \pm 6.8(\mathrm{p}<0.025$, nonimmunized compared with fibroblast /IL-2-treated mice).

tion was seen in the brains of the treated animals. It was composed primarily of CD8+ and CD4+ T cells, suggesting a specific immunocytotoxic antitumor response.

\section{ALLOGENEIC IMMUNOGENE THERAPY}

The use of genetically engineered allogeneic cells as a delivery vehicle for cytokines may be an attractive alternative method of cytokine therapy. In our laboratory, we introduced the gene for IL-2 into a mouse fibroblast cell line (LM) expressing defined MHC determinants $\left(\mathrm{H}-2^{\mathrm{k}}\right)$. We tested the immunotherapeutic properties of these allogeneic IL-2-secreting cells in C57Bl/6 mice harboring G1261 glioma in both subcutaneous and intracerebral models. ${ }^{14,15,24}$ We found that in mice with an intracerebral malignant glioma treated by injection into the tumor bed of allogeneic fibroblasts genetically modified to secrete IL-2, survival was significantly prolonged compared with that of untreated control mice injected with glioma cells alone (Fig. 1). ${ }^{15,24}$

Furthermore, using a ${ }^{51} \mathrm{Cr}$ release cytotoxicity assay, a significant and specific antiglioma immunocytotoxic response was detected in the animals treated with the allogeneic IL-2-secreting fibroblasts (Table 1). The intracranial implantation of the modified fibroblasts was shown to be without detectable toxicity, and the animals exhibited no neurological deficit.
TABLE 1

The effect of monoclonal antibodies against T-cell subsets or natural killer/lymphokine-activated killer cells on the antiglioma cytotoxic activities of spleen cells obtained from mice injected intracerebrally with a mixture of glioma and cytokine(s)-secreting cells*

\begin{tabular}{llc}
\hline \hline & & \\
Cell Types for & Cytolysis at \\
Immunization & Treatment & E:T \\
glioma & - & Ratio of $100: 1(\%)$ \\
& anti-Lyt-2.2 & $3.6 \pm 1.2$ \\
glioma + LM & anti-asialo GM1 & $-1.4 \pm 2.5$ \\
& - & $-7.1 \pm 2.8$ \\
glioma + LM-IL-2 & anti-Lyt-2.2 & $5.8 \pm 2.8$ \\
& anti-asialo GM1 & $-1.9 \pm 4.2$ \\
& - & $-7.8 \pm 1.8$ \\
& anti-Lyt-2.2 & $5.2 \pm 2.7 \dagger$ \\
& anti-asialo GM1 & $-6.6 \pm 2.3$ \\
\hline
\end{tabular}

* The C57BL/6 mice received a single intracerebral injection of $\left(10^{5}\right)$ glioma cells together with one of the modified fibroblast cell types $\left(10^{6}\right.$ cells). Three weeks postinjection, mononuclear cells from the spleens of the immunized mice were used for the ${ }^{51} \mathrm{Cr}$ release assay. All values represent the mean \pm standard deviation of triplicate determinations. Abbreviations: $L M=$ mouse fibroblast cell line.

$\dagger \mathrm{p}<0.005$ relative to ${ }^{51} \mathrm{Cr}$ release for spleen cells obtained from mice immunized with glioma.

$\ddagger \mathrm{p}<0.05$ relative to ${ }^{51} \mathrm{Cr}$ release for spleen cells obtained from mice immunized with glioma + mouse fibroblast cells. 

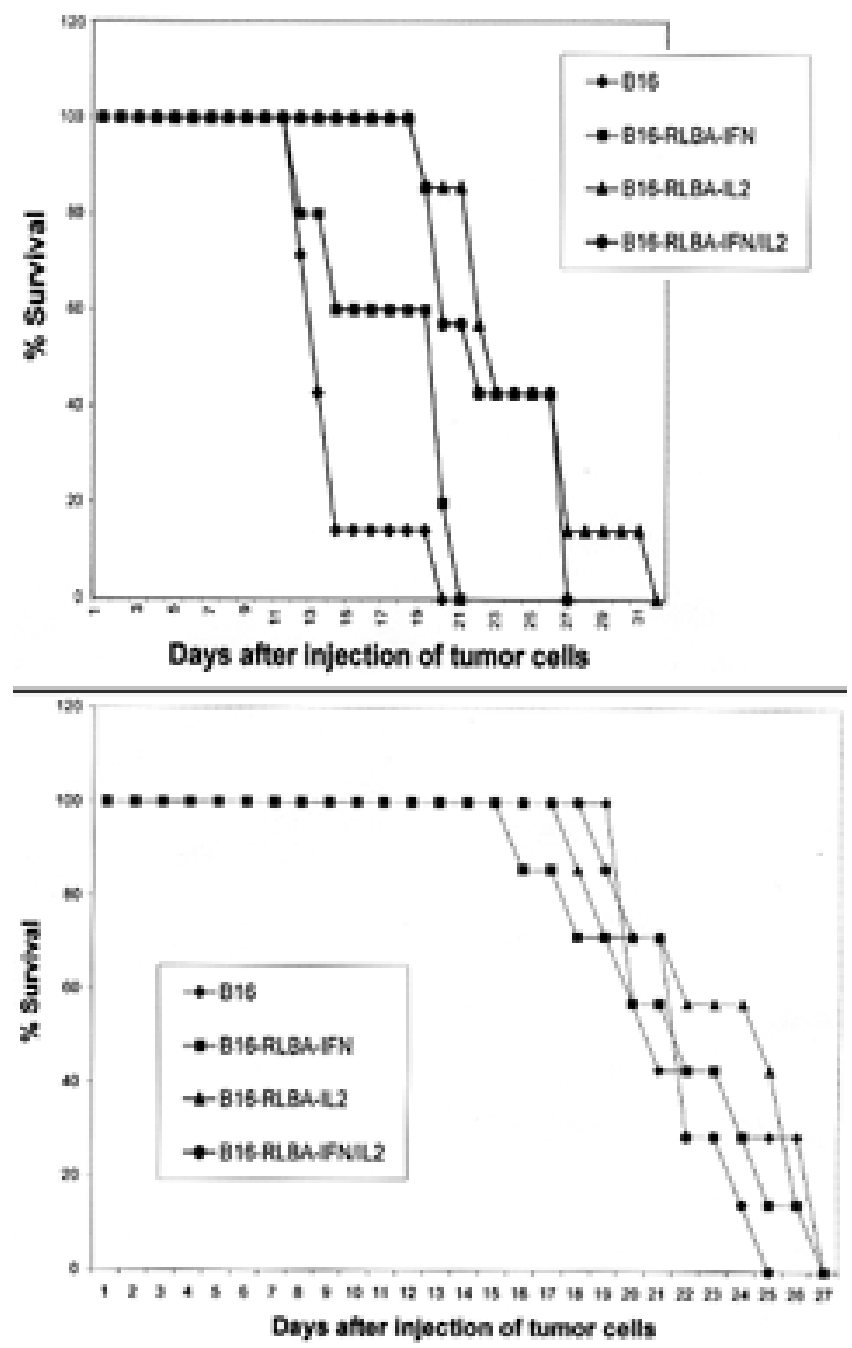

Fig. 2. A: Graph showing the survival of mice injected intracerebrally with a mixture of B16F1 melanoma cells (RLBA-IL-2 cells. The RLBA cells are fibroblasts transfected with genomic DNA from B16 melanoma cells and selected for the expression of melanoma-associated antigens). The $\mathrm{C} 57 \mathrm{~B} 1 / 6$ mice were injected intracerebrally with a mixture of B16F1 melanoma cells $\left(10^{3}\right)$ and one of the cell types $\left(10^{6}\right)$. Mean survival times ( \pm standard deviation) in days were as follows: B16 cells alone, $14 \pm 2.6$; B16 + RLBA-IFN- $\gamma$ cells, $17.4 \pm 3.7$; B16 + RLBA-IL-2 cells, $24.6 \pm$ 4.0; B16 + RLBA-IFN $\gamma / \mathrm{IL}-2$ cells, $23.1 \pm 3.4$ (p $<0.005$, nonimmunized or RLBA-IFN $\gamma$ compared with RLBA-IL-2; $\mathrm{p}<$ 0.005 ; nonimmunized or RLBA-IFN $\gamma$ compared with RLBAIFN $\gamma /$ IL-2. B: Graph showing the survival of mice injected intracerebrally with $\mathrm{B} 16 \mathrm{~F} 1$ melanoma cells and subcutaneously with cytokine-secreting cells. The mice were injected intracerebrally with B16F1 cells $\left(10^{3}\right)$ and subcutanously with one of the cell types ( $10^{6}$ cells). Mean survival time in days ( \pm standard deviation): B16 cells alone, $22.7 \pm 3$; B16 + RLBA-IFN $\gamma, 21.7 \pm 3.6$; B16 + RLBA-IL-2, $23.3 \pm 3.4$; B16 + RLBA-IFN $\gamma / \mathrm{IL}-2,22 \pm 1.9$.

Based on these experiments, we extended our studies to the treatment of intracerebral metastatic cancer. In prior studies, immunity to melanoma and prolongation of survival was seen in mice with peripheral melanoma that were treated with allogeneic fibroblasts genetically engi- neered to secrete cytokines. ${ }^{22,23}$ We found that in mice with an intracerebral malignant melanoma in which allogeneic fibroblasts genetically modified to secrete IL-2 were injected into the tumor bed, survival was significantly longer than that in untreated control mice injected with melanoma cells alone. ${ }^{15}$ In these experiments, IL-2-secreting fibroblasts were cotransfected with genomic DNA from $\mathrm{B} 16 \mathrm{~F} 1$ melanoma cells to generate fibroblasts that secrete IL-2 and express melanoma-associated antigens (RLBA-IL-2). Specific and significant antimelanoma immunocytotoxic responses were found in mice with intracerebral melanoma treated with either an intracerebral or subcutaneous injection of RLBA-IL-2 cells, although prolongation of survival was only demonstrated in the animals that received an intracerebral injection of the treatment cells. Like other allografts, the cells were eventually rejected, preventing the possible growth of the vaccine itself in the tumor-bearing mice (Fig. 2).

We next investigated whether an intracerebral metastatic breast tumor can be treated successfully with this cellular vaccine when allogeneic fibroblasts are used as a novel vehicle for the delivery of IL-2. Using the EO771 breast cancer cell line-a spontaneously arising breast cancer tumor produced in C57BL/6 mice-we intracerebrally injected $10^{5} \mathrm{EO} 771$ cells into the right frontal lobe along with a single intratumoral injection of $10^{6}$ allogeneic fibroblasts genetically engineered to secrete IL-2. The allogeneic fibroblasts transfected with the $I L-2$ gene formed large quantities of IL-2 as measured by an enzyme-linked immunosorbent assay. Groups of control mice were treated with either: 1) allogeneic fibroblasts transfected with the retroviral vector but not the $I L-2$ gene; or 2) media. We found a significant prolongation $(\mathrm{p}<$ 0.005 ) of survival in animals with intracerebral metastatic breast cancer that were treated with IL-2-secreting allogeneic fibroblasts only. Four of 10 mice in the IL-2-treated group did not develop tumors and were long-term survivors. These mice underwent intracerebral tumor rechallenge at 90 days but without treatment cells, and the findings were compared with those obtained in four naïve mice receiving intracerebral tumor. Again, the animals that previously had been treated with IL-2-secreting fibroblasts had a markedly prolonged survival $(p<0.05)$ compared with control mice following a second challenge with tumor cells; this result suggested the production of "immune memory." This work represents a new treatment for breast cancer metastatic to the brain in which we use a cellular vaccine consisting of allogeneic fibroblasts genetically engineered to secrete cytokines as a novel means for delivery of immunogene therapy; further, it demonstrates the induction of long-term immunity against tumor (Fig. 3).

\section{ALTERATION OF MHC: ALLOGENEIC COMPARED WITH SYNGENEIC}

The decision to use allogeneic rather than syngeneic cells in the cellular vaccine was based on evidence that allogeneic MHC determinants augmented the immunogenic properties of the tumor vaccine. , $12,17,19,30$ Hammerling, et al., ${ }^{17}$ transfected genes for allogeneic MHC antigens into mouse fibrosarcoma cells, and the cells; immuno- 


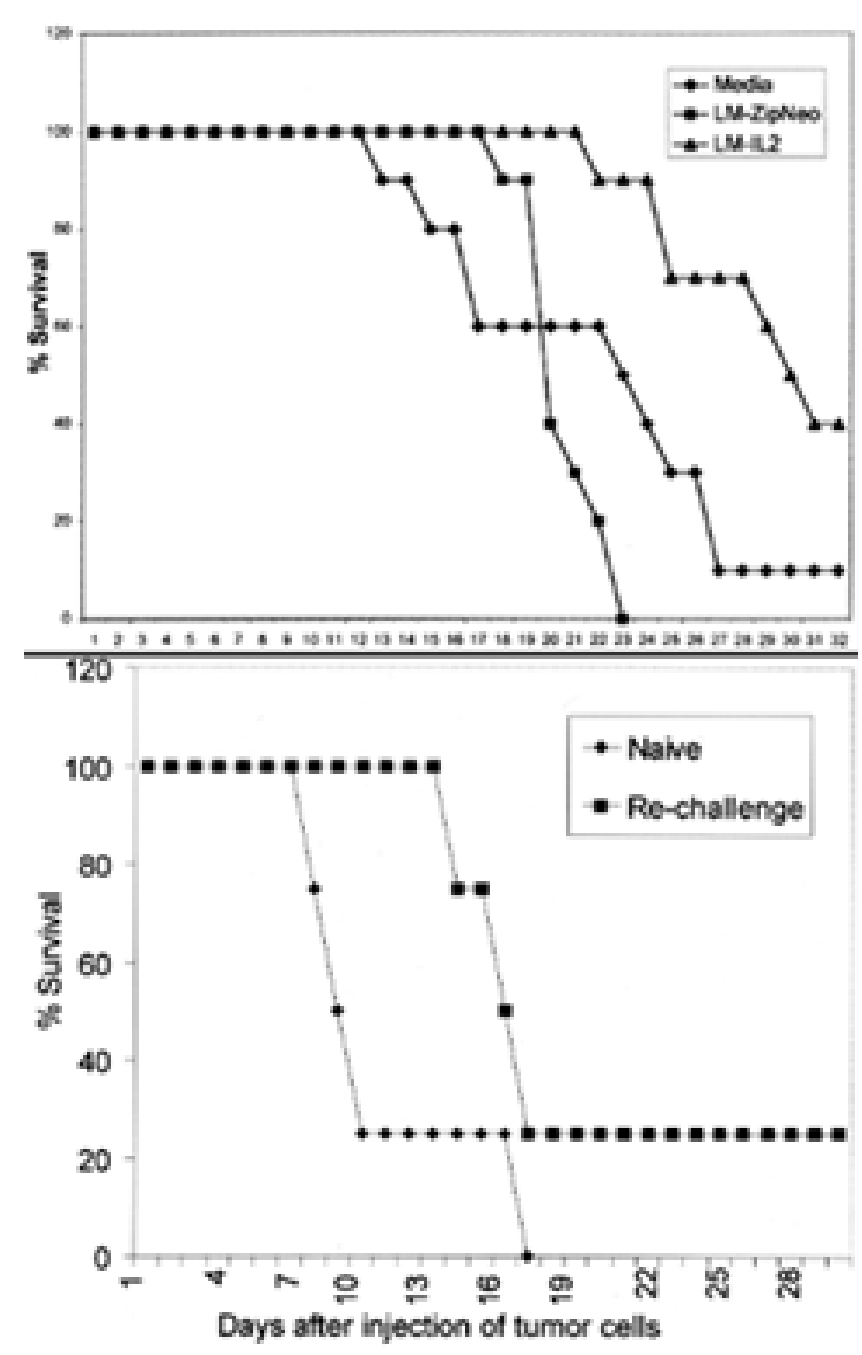

Fig. 3. A: Graph showing the survival rate of mice injected intracerebrally with a mixture of breast carcinoma cells and fibroblasts (LM cells) engineered to secrete IL-2. The C57Bl/6 mice (10 per group) were injected intracerebrally with a mixture of $5 \times 10^{4}$ EO771 breast carcinoma cells grown in vitro and 106 of one of the cell types. The median lengths of survival in days ( \pm standard deviation) were as follows: EO771 alone, $22 \pm 5.9$; EO771 plus LM-ZipNeo cells, $20.7 \pm 1.5$; EO771 plus LM-IL-2 cells, $29 \pm$ 3.5. Nonimmunized or EO771 treated with nonsecreting neomycin-resistant cells compared with LM-IL-2, p < 0.005. B: Graph showing survival of $\mathrm{C} 57 \mathrm{~B} 1 / 6$ mice that survived a prior injection of EO771 cells and LM-IL-2 fibroblasts injected with EO771 cells alone. Four mice surviving 90 days after the prior injection of EO771 cells and LM-IL-2 fibroblasts were injected intracerebrally a second time with $5 \times 10^{4}$ EO771 cells alone. As a control, four naïve $\mathrm{C} 57 \mathrm{Bl} / 6$ mice were injected intracerebrally with an equivalent number of EO771 cells. Median survival time ( \pm standard deviation) in days: naïve animals, $11 \pm 4.1$; rechallenged animals, $18.8 \pm 6.3(\mathrm{p}<0.05$ for difference in survival).

genic properties were increased as a result. Hui, et al., ${ }^{19}$ found that immunization with a $\mathrm{k}$ haplotype tumor modified by introducing the gene of $\mathrm{H}-2 \mathrm{~K}^{\mathrm{b}}$ led to an antitumor cellular immune response in $\mathrm{k}$ haplotype mice. OstrandRosenberg, et al., ${ }^{30}$ Fearon, et al., ${ }^{9}$ and Gattoni-Celli, et al., ${ }^{12}$ have reported analogous results.
Because the fibroblasts used in our previous studies were allogeneic in $\mathrm{C} 57 \mathrm{Bl} / 6$ mice, presentation of tumorassociated antigens may have followed antigen uptake by antigen-presenting cells of the host with the tumor antigens processed and presented to cytotoxic $\mathrm{T}$ cells of the host. Conceivably, the immunogenic properties of the allogeneic cells would be enhanced if the fibroblasts were modified to form syngeneic $\mathrm{H}-2 \mathrm{~K}^{\mathrm{b}}$ Class I determinants. The self MHC Class I determinants might provide a mechanism for the direct presentation of antigenic peptides to cytotoxic $\mathrm{T}$ lymphocytes of the tumor-bearing host, further enhancing the cells' immunogenic properties.

To investigate this question, a plasmid (pBR327H-2K $\left.{ }^{b}\right)$ was used to introduce expression-competent genes for $\mathrm{H}$ $2 \mathrm{~K}^{\mathrm{b}}$ determinants into the fibroblasts. In initial studies, we found that intratumoral administration of a cellular vaccine, consisting of fibroblasts that expressed both syngeneic and allogeneic MHC determinants, prolonged survival of mice with either subcutaneous gliomas or melanoma and that it induced an antitumor immune response. ${ }^{8,13}$ We found a further prolongation of survival, complete tumor regression in some cases, and an increased immunocytotoxic response in mice with intracerebral gliomas treated with this allogeneic/syngeneic vaccine. ${ }^{13}$

Two possible mechanisms may explain the immune response of mice treated with the allogeneic/syngeneic cells. Large numbers of cytotoxic T lymphocytes with specificity toward tumor-associated antigens may have been generated in the microenvironment of allograft recognition and rejection. The immunogenic properties of tumor cells transfected with genes specifying allogeneic determinants are supportive of the interpretation. In addition, MHC Class I genes that share identity with the tumor-bearing host may present tumor-associated T-cell epitopes directly to cytotoxic T lymphocytes. Fibroblasts can act as efficient antigen-presenting cells; ${ }^{3,40}$ they form B.7.1, a costimulatory molecule required for antigen-specific T-cell activation, as well as MHC Class I determinants. Our antibody depletion ${ }^{51}$ chromium release studies demonstrated that a specific CD8+ immunocytotoxic response against glioma was stimulated by immunization with the allogeneic/syngeneic IL-2-secreting cells. Thus, our studies demonstrated that the immune response could be augmented by genetic modification of one cell to form syngeneic $\mathrm{MHC}$ and to secrete immune-augmenting cytokines.

The genetic modification of a cell line rather than cells from a primary neoplasm has other important advantages for use in tumor treatment. Modification of neoplastic cells obtained directly from tumor-bearing patients may be difficult. A primary tumor cell line, required for retroviral modification, has to be established. The establishment of a cell line from a primary neoplasm is not always possible. Furthermore, it is conceivable that a subpopulation of the primary tumor, selected for its capacity to grow in vitro, may not reflect the tumor cell population as a whole, especially because tumors such as glioma are known to be heterogeneous. In addition, the use of tumor cells as the vehicle of cytokine delivery is of concern because the cells themselves may grow into a tumor. Thus, the advantage of using an allogeneic fibroblast cell line 


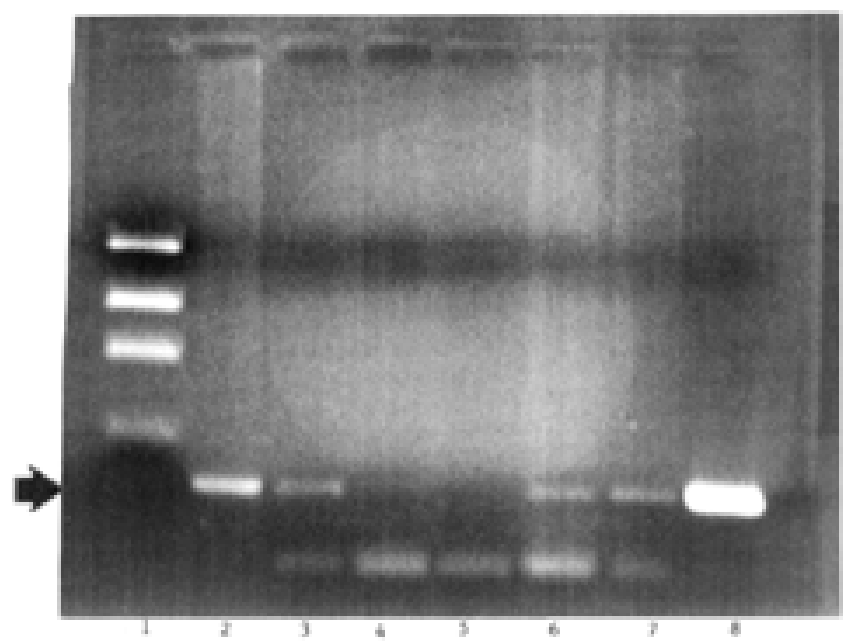

Fig. 4. Polymerase chain reaction anaylsis for the survival of modified fibroblasts in the CNS. The analysis was performed to detect the presence of the neomycin resistance gene in brain sections taken at various time intervals (0-60 days) after implantation of modified fibroblasts into the CNS in allogeneic and syngeneic mice. Sequences of DNA for the neomycin resistance gene were observed on Days 8 and 14 but not on Day 28 or 60 after implantation in allogeneic mice, and up to 55 days in syngeneic mice. Lane 1, low-mass molecular marker; Lane 2, 8 days after injection into allogeneic mice; Lane 3, 14 days after injection into allogeneic mice; Lane 4, 28 days after injection into allogeneic mice; Lane 5,60 days after injection into allogeneic mice; Lane 6, 55 days after injection into syngeneic mice; Lane $7,10^{3}$ LM-IL-2 cells; Lane 8, pZipNeo plasmid. Arrow indicates the location of the $249 \mathrm{bp}$ Neo $o^{r}$ gene.

is that it is reliable, readily available, stably transfected, and not tumorigenic. Like other allografts, the cells are rejected. Furthermore, the number of cells can be expanded, as desired, for multiple rounds of therapy. Additionally the slow, continuous release of cytokines and the eventual rejection of the allograft may be a useful advantage in the treatment of brain tumor cases in which long-term secretion of high concentrations of certain cytokines may be associated with increased morbidity and mortality rates.

\section{TOXICITY AND SURVIVAL OF A CELLULAR VACCINE}

The toxic effects of cytokines in the CNS may limit the quantity that can be administered. ${ }^{4,21,36}$ Neurological effects have been demonstrated in animals injected intracranially with syngeneic cytokine-secreting cells. The coimplantation into the rat brain of syngeneic glioma cells and synergeneic cells modified by retroviral transduction to secrete IL-2 or IFN $\gamma$ has been shown to result in shortterm cell-mediated antiglioma responses. However, the survival of the tumor-bearing rats was not prolonged, ${ }^{42}$ and the animals died of secondary effects including severe cerebral edema. Most of the systemic toxicities of IL-2 therapy can be avoided by the introduction of the gene for IL-2 directly into the tumor mass, resulting in high local concentrations of the cytokine. This form of treatment is particularly advantageous in primary gliomas, be-

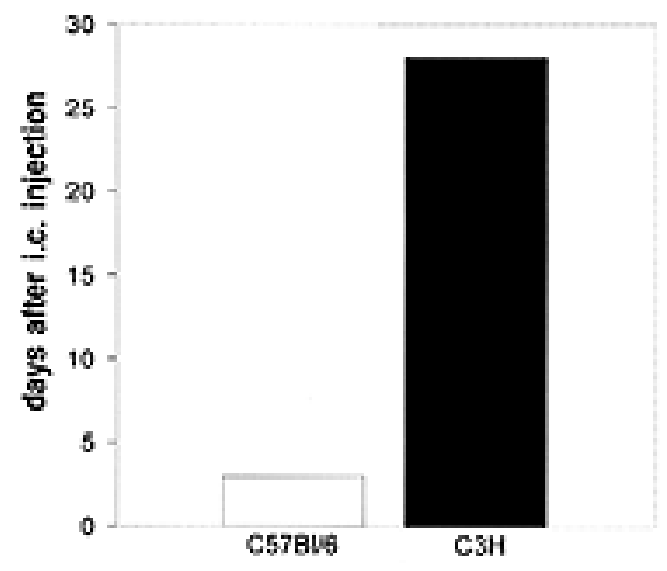

Fig. 5. Bar graph showing in vitro recovery analysis for the survival of modified fibroblasts in the CNS. The recovery of modified fibroblasts in cultures derived from brain sections of the treated mice obtained at various time intervals after implantation (0-60 days) in allogeneic $(\mathrm{C} 57 \mathrm{Bl} / 6)$ and syngeneic $(\mathrm{C} 3 \mathrm{H})$ mice grown in the presence of neomycin. Allogeneic cells could be recovered for only up to 3 days after intracerebral injection implantation, whereas syngeneic cells were recovered for up to 28 days after implantation.

cause these tumors usually only recur locally and are rarely metastatic.

The toxicity of a cellular-based cytokine gene therapy for tumors is likely to depend in part on the survival of the genetically modified cells in the CNS. We investigated the survival of an allogeneic IL-2 secreting vaccine in the CNS by two different means. First, polymerase chain reaction analysis was used to detect the presence of the neomycin resistance gene, a component of the modified retrovirus used to modify the cells for IL-2-secretion, in brain sections at various time intervals after implantation (Fig. 4).

As a second means of assessing the survival of the modified cells in the CNS, we attempted to recover the modified fibroblasts from cultures that were derived from brain sections obtained at various time intervals after implantation. Results of both assays indicated that the period of survival of allogeneic cells in the CNS was less than 28 days (Fig. 5). Presumably the cells, like other allografts, were rejected. The cells were well tolerated, and no significant neurological toxicity was demonstrated in the animals. ${ }^{16}$ This finding suggests that cytokine-secreting allogeneic cells may serve as a useful vehicle for the safe delivery of cytokines into brain neoplasms, and it supports the possibility and safety of using a monthly retreatment schedule in a clinical protocol. Most of the systemic toxicities associated with IL-2 therapy may be avoided by introducing the cytokine gene for IL-2 directly into the tumor mass, resulting in lower local concentrations of the cytokine.

\section{ROUTE OF DELIVERY}

Another problem related to gene therapy for CNS tumors has been the determination of the most optimum route of delivery. In recent studies, we compared the effect 
of direct intracerebral with systemic administration of a tumor vaccine consisting of allogeneic fibroblasts genetically engineered to secrete cytokines; the results obtained using the two injection methods were compared for survival and immune response in mice with glioma or melanoma. ${ }^{14}$ We found that only direct intracerebral injection into the tumor bed resulted in significantly prolonging survival, despite a significantly elevated systemic immunocytotoxic response generated against the tumor antigens by ${ }^{51} \mathrm{Cr}$ release assay in both the subcutaneously and intracerebrally immunized animals. ${ }^{14}$ Subcutaneous administration of IL-2-secreting cells in addition to tumor cells was not effective in the treatment of mice with intracerebral glioma or melanoma. ${ }^{14}$ This result was somewhat surprising because we observed a significantly elevated systemic immunocytotoxic response by ${ }^{51} \mathrm{Cr}$ release assay in the subcutaneously immunized animals. Thus immunization by subcutaneous injection was not as effective as injection of the vaccine directly into the tumor bed itself.

One of the major concerns related to the immunological treatment of brain tumors is the effect of the blood-brain barrier on the development of a host immune response in the CNS. Studies in which investigators implanted IL4-secreting plasmacytoma cells into the brains of nude mice, in addition to human glioma cells, have demonstrated a dramatic eosinophilic infiltrate in regions of necrotic tumor; this finding suggests that an immune response can occur against a tumor of CNS in situ. The response, however, was non-T cell dependent. ${ }^{45} \mathrm{We}$ found that a specific and significant systemic immunocytotoxic response (as measured by a ${ }^{51}$ chromium release assay) was present in mice with an intracerebral glioma treated with allogeneic IL-2-secreting fibroblasts administered directly into the tumor. ${ }^{15,24}$ Thus, the secretion of IL-2 by the cellular immunogen ${ }^{44,46}$ or an immunogenic derivative of the cells may have altered the blood-brain barrier, enabling the immunogen to reach the spleen and lymph nodes in the periphery. Furthermore, in a recent report the investigator showed that subcutaneous injection of irradiated GMCSF-transduced glioma cells can induce a potent immune response to intracranial gliomas, both as a vaccination against subsequent intracranial glioma cell implantation and for treatment of established intracranial glioma. ${ }^{18}$

\section{CHOICE OF CYTOKINES}

In addition to investigating the optimum route of delivery, the most efficacious combination of cytokines is currently being examined. Several laboratories have recently reported their results using GM-CSF alone ${ }^{18}$ or in combination with IL-2. ${ }^{32}$ Subcutaneous injection of irradiated GM-CSF-transduced glioma cells induced a potent immune response to intracranial gliomas both as a vaccination against subsequent intracranial glioma cell implantation and for treatment of established intracranial glioma. ${ }^{18}$ In addition, the combination of IL-2 injected intracerebrally and GM-CSF injected subcutaneously was synergistic. ${ }^{41}$ Thus these studies and others suggest that tumor vaccines consisting of tumor antigens in the presence of cytokines may be effective in the treatment of CNS tumors both as a systemic vaccine and administered locally. Certainly the most effective cytokine for the treatment of glioma has not been determined.
We have also investigated whether the use of multiple cytokines may be synergistic. We previously reported that the best antitumor response occurred when fibroblasts were transfected with the genes for both IL-2 and IFN $\gamma .{ }^{24}$ Upon injection of these double cytokine-secreting cells into the tumor bed, there was a markedly prolonged increase in survival time as well as the development of a strong antitumor immunocytotoxic response as measured by a standard ${ }^{51} \mathrm{Cr}$ release assay. Interleukin- 12 has also been reported to be effective in stimulating an antitumor immune response and subsequent prolongation in survival in animals harboring intracerebral tumors. We investigated the effectiveness of using allogeneic fibroblasts genetically engineered to secrete IL-12 alone or in combination with cells engineered to secrete both IL-12 and IL-2 in the treatment of $\mathrm{C} 57 \mathrm{Bl} / 6$ mice harboring an intracerebral glioma (Gl261). Cytokine secretion was confirmed by an enzyme-linked immunosorbent assay. We found no prolongation of survival in animals treated with IL-12-secreting fibroblasts alone. However, a markedly prolonged increase in survival $(p<0.05)$ was demonstrated in the mice with intracerebral glioma treated with fibroblasts secreting both IL-12 and IL-2 in comparison with control animals in which the glioma was treated with nonsecreting fibroblasts or with media. We also noted a prolongation in survival in the mice treated with IL-2-secreting fibroblasts, although the most dramatic response was noted in those treated with fibroblasts secreting both IL-2 and IL-12. In conclusion these data suggest that allogeneic fibroblasts genetically engineered to secrete both IL-2 and IL-12 are effective in the treatment of intracerebral glioma, but IL-12-secreting fibroblasts alone were not shown to prolong survival in these preliminary studies.

\section{TISSUE-SPECIFIC PROMOTORS}

Another challenge for gene therapy is that of tissue specificity and selectivity. Retroviruses are selective as they integrate only into dividing cells, whereas adenoviruses may be advantageous because they can infect nondividing cells. The use of cell type-specific enhancers may add to the delivery efficacy of these viruses as well as their specificity. Because glial fibrillary acidic protein is specific for cells of glial origin and because the majority of primary brain tumors are of glial origin, this is one candidate gene for enhancing tissue specifity. Using genetic engineering techniques, a replication-incompetent adenovirus was constructed containing a fragment enhancer region of the GFAP gene coupled to a tetracycline transactivator. ${ }^{6}$ This produced a delivery system that is glial specific and repressible. This type of novel virus delivery system should be useful for a variety of gene therapy approaches for CNS tumors, including the delivery of cytokines, and we enthusiastically await further studies.

\section{CONCLUSIONS}

Based on studies conducted in our laboratory as well as the results reported by other investigators, it is likely that immunotherapy with cytokine-secreting tumor vaccines in combination with traditional surgery, radiotherapy, and chemotherapy would provide improved treatment in eliminating a greater proportion of the tumor cell population 
and prolonging life for patients with malignant intracerebral tumors. Tumor vaccines in which cells are genetically engineered to secrete cytokines and other immunoregulatory molecules as a means of targeted immunogene therapy are one of the most promising new treatments for intracerebral malignant tumors.

\section{References}

1. Andreansky S, He B, van Cott J, et al: Treatment of intracranial gliomas in immunocompetent mice using herpes simplex viruses that express murine interleukins. Gene Ther 5:121-130, 1998

2. Barba D, Saris SC, Holder C, et al: Intratumoral LAK cell and interleukin-2 therapy of human gliomas. J Neurosurg 70: 175-182, 1989

3. Battegay M, Lother H, Gessner A, et al: Fibroblasts as efficient antigen-presenting cells in lumphoid organs. Science 268: 1343-1345, 1995

4. Birchfield GR, Rodgers GM, Girodias KW, et al: Hypoprothrobinemia associated with interleukin-2 therapy. J Immunother 11:71-75, 1992

5. Borden EC, Sondel PM: Lymphokines and cytokines as cancer treatment. Immunotherapy realized. Cancer 65 (Suppl 3): 800-814, 1990

6. Chen J, Bezdek T, Chang J, et al: A glial-specific, repressible, adenovirus vector for brain tumor gene therapy. Cancer Res 58:3504-3507, 1998

7. Cook AW, Carter WA, Nidzgorski R, et al: Human brain tumorderived cell lines: growth rate reduced by human fibroblast interferon. Science 219:881-883, 1983

8. DeZoeten EF, Carr-Brendel V, Cohen EP: Resistance to melanoma in mice immunized with semiallogeneic fibroblasts transfected with DNA from mouse melanoma cells. J Immunol 160:2915-2922, 1998

9. Fearon ER, Itaya T, Hunt B, et al: Induction in a murine tumor of immunogenic tumor variants by transfection with a foreign gene. Cancer Res 48:2975-2980, 1988

10. Gabrilove JL, Jakubowski A: Hematopoietic growth factors: biology and clinical application. Monogr J Natl Cancer Inst 10:73-77, 1990

11. Gandolfi L, Solmi L, Pizza GC, et al: Intratumoral echo-guided injection of interleukin-2 and cytokine-activated killer cells in hepatocellular carcinoma. Hepatogastroenterology 36:352-356, 1989

12. Gattoni-Celli S, Willett CG, Rhoads DB, et al: Partial suppression of anchorage-independent growth and tumorigenicity in immunodeficient mice by transfection of the H-2 class I gene $\mathrm{H}-2 \mathrm{~L}^{\mathrm{d}}$ into a human colon cancer cell line (HCT). Proc Natl Acad Sci USA 85:8543-8547, 1988

13. Glick RP, Lichtor T, DeZoeten E, et al: Prolongation of survival in mice with intracerebral glioma treated with semi-allogeneic/syngeneic fibroblasts. J Neurosurg 88:388-389, 1998 (Abstract)

14. Glick RP, Lichtor T, Kim TS, et al: Fibroblasts genetically engineered to secrete cytokines suppress tumor growth and induce antitumor immunity to a murine glioma in vivo. Neurosurgery 36:548-555, 1995

15. Glick RP, Lichtor T, Mogharbel A, et al: Intracerebral versus subcutaneous immunization with allogeneic fibroblasts genetically engineered to secrete interleukin- 2 in the treatment of central nervous system glioma and melanoma. Neurosurgery 41: 898-907, 1997

16. Griffitt W, Glick RP, Lichtor T, et al: Survival and toxicity of an allogeneic cytokine-secreting fibroblast vaccine in the central nervous system. Neurosurgery 42:335-340, 1998

17. Hammerling GJ, Klar D, Katzav S, et al: Manipulation of met- astasis and tumour growth by transfection with histocompatibility class I genes. J Immunogen 13:153-157, 1986

18. Herrlinger U, Kramm CM, Johnston KM, et al: Vaccination for experimental gliomas using GM-CSF-transduced glioma cells. Cancer Gene Ther 4:345-352, 1997

19. Hui KM, Sim TF, Foo TT, et al: Tumor rejection mediated by transfection with allogeneic class I histocompatibility gene. J Immunol 143:3835-3843, 1989

20. Kelso A: Cytokines: structure function and synthesis. Curr Opin Immunol 2:215-225, 1989

21. Kim H, Rosenberg SA, Steinberg SM, et al: A randomized double blind comparison of the antiemetic efficacy of ondansetron and dropidol in patients receiving high dose interleukin-2. J Immunother Emphasis Tumor Immunol 16:60-65, 1994

22. Kim TS, Russell SJ, Collins MKL, et al: Immunity to B16 melanoma in mice immunized with IL-2-secreting allogeneic mouse fibroblasts expressing melanoma-associated antigens. Int J Cancer 51:283-289, 1992

23. Kim TS, Xu WS, Cohen EP: Immunization with interleukin$2 /$ interferon- $\gamma$ double cytokine-secreting allogeneic fibroblasts prolongs the survival of mice with melanoma. Melanoma Res 5:217-227, 1995

24. Lichtor T, Glick RP, Kim TS, et al: Prolonged survival of mice with glioma injected intracerebrally with double cytokine-secreting cells. J Neurosurg 83:1038-1044, 1995

25. Lotze MT, Chang AE, Seipp CA, et al: High-dose recombinant interleukin-2 in the treatment of patients with disseminated cancer: responses, treatment-related morbidity and histologic findings. JAMA 256:3117-3124, 1986

26. Mahaley MS, Mettlin C, Natarajan N, et al: National survey of patterns of care for brain-tumor patients. J Neurosurg 71: 826-836, 1989

27. Merchant RE, Ellison MD, Young HF: Immunotherapy for malignant glioma using human recombinant interleukin-2 and activated autologous lymphocytes. A review of pre-clinical and clinical investigations. J Neurooncol 8:173-188, 1990

28. Merchant RE, McVicar DW, Merchant LH, et al: Treatment of recurrent malignant glioma by repeated intracerebral injections of human recombinant interleukin-2 alone or in combination with systemic interferon- $\alpha$. Results of a phase I clinical trial. J Neurooncol 12:75-3, 1992

29. Mizuno M, Yoshida J, Takaoka T, et al: Liposomal transfection of human gamma-interferon gene into human glioma cells and adoptive immunotherapy using lymphokine-activated killer cells. J Neurosurg 80:510-514, 1994

30. Ostrand-Rosenberg S, Thakur A, Clements V: Rejection of mouse sarcoma cells after transfection of MHC class II genes. J Immunol 144:4068-4071, 1990

31. Pizza G, Viza D, DeVince C, et al: Intralymphatic administration of interleukin-2 (IL-2) in cancer patients: a pilot study. Cytokine Res 7:45-48, 1988

32. Ram Z, Walbridge SS, Heiss JD, et al: In vivo transfer of the human interleukin-2 gene: negative tumoricidal results in experimental brain tumors. J Neurosurg 80:535-540, 1994

33. Robinson WA, Jobe K, Stevens R: Central nervous system metastases in malignant melanoma. Cancer Treat Res 35: 155-163, 1987

34. Rosenberg SA, Lotze MT, Muul LM, et al: A progress report on the treatment of 157 patients with advanced cancer using lymphokine-activated killer cells and interleukin-2 or high-dose interleukin-2 alone. N Engl J Med 316:889-897, 1987

35. Rosenberg SA, Lotze MT, Mule JJ: New approaches to the immunotherapy of cancer. Ann Intern Med 108:853-864, 1988

36. Rosenberg SA, Yang JC, Topalian LS, et al: Treatment of 283 consecutive patients with metastatic melanoma or renal cell cancer using high-dose bolus interleukin 2. JAMA 271: 907-913, 1994

37. Roszman T, Elliot L, Brooks W: Modulation of T-cell function by gliomas. Immunol Today 12:370-374, 1991 


\section{Cytokine immunogene therapy}

38. Sama G, Collins J, Figlin R, et al: A pilot study of intralymphatic interleukin-2. II Clinical and biological effects. J Biol Response Modif 9:81-86, 1990

39. Saris SC, Spiess P, Lieberman DM, et al: Treatment of murine primary brain tumors with systemic interleukin-2 and tumorinfiltrating lymphocytes. J Neurosurg 76:513-519, 1992

40. Schoenberger SP, Jonges LE, Mooijaart RJD, et al: Efficient direct priming of tumor-specific cytotoxic $\mathrm{T}$ lumphocytes in vivo by an engineered APC. Cancer Res 58:3094-3100, 1998

41. Thompson RC, Pardoll DM, Jaffee EM, et al: Systemic and local paracrine cytokine therapies using transduced tumor cells are synergistic in treating intracranial tumors. J Immunother Emphasis Tumor Immunol 19:405-413, 1996

42. Tjuvajev J, Gansbacher B, Desai R, et al: RG-2 glioma growth attenuation and severe brain edema caused by local production of interleukin-2 and interferon. Cancer Res 55:1902-1910, 1995

43. Vaquero J, Martinez R, Oya S, et al: Intratumoral injection of autologous lymphocytes plus human lymphoblastoid interferon for the treatment of glioblastoma. Acta Neurochir 98:35-41, 1989
44. Watts RG, Wright JL, Atkinson LL, et al: Histopathological and blood-brain barrier changes in rats induced by intracerebral injection of human recombinant interleukin 2. Neurosurgery 25: 202-208, 1989

45. Yu JS, Wei MX, Chicca A, et al: Treatment of glioma by engineered interleukin-4-secreting cells. Cancer Res 53: 3125-3128, 1993

46. Zhang RD, Price JE, Fujimaki T, et al: Differential permeability of the blood brain barrier in experimental brain metastases produced by human neoplasms implanted into nude mice. Am J Pathol 141:1115-1124, 1992

Manuscript received November 8, 2000.

Accepted in final form November 27, 2000.

Address reprint requests to: Roberta P. Glick, M.D., Department of Neurosurgery, Cook County Hospital, 1835 West Harrison, Chicago, Illinois 60612. 